\title{
Comparative Analysis of Optimal Temperature Distributions in the Responsible Sections of Load Bearing Structures
}

\author{
Mikhail Yu. Livshits ${ }^{1,}{ }^{*}$, Boris B. Borodulin ${ }^{1}$ \\ ${ }^{1}$ Samara State Technical University, 443000 Samara, Russia
}

\begin{abstract}
This work presents a comparative analysis of the temperature distribution for different thermal conductivity coefficients. The analysis of the general problem shows that temperature distribution in the critical sections of the load bearing structures significantly affects of the diagnostic instrumentation's data accuracy placed on them. Temperature distribution control optimization allows to reduce influence of temperature factors on the accuracy of diagnostic instrumentation. All this requires a thorough analysis.
\end{abstract}

\section{Problem statement}

The temperature distribution in the critical sections of the load bearing structures significantly affects the accuracy of diagnostic instrumentation data placed on them, especially the opto-mechanical equipment. In [1] for a load bearing structure in the form of a rectangular prism is justified of the optimal accuracy of maintaining the required temperature $T_{t a r}$ and speed of operation nonsmooth boundary value problems. Taking into account the specific operation of the optical equipment placed on the supporting structure can be modified to spatially one-dimensional nonsmooth boundary value problems.

\section{Numerical calculations}

Semi-infinite optimization on the line in a given section $l_{y}{ }^{*} \in l_{y}, l_{y}{ }^{*}=$ const $; l_{z}{ }^{*} \in l_{z}$, $l_{z}^{*}=$ const $;$ in relative $\left(l_{x}, l_{y}, l_{z}\right)$ Cartesian coordinates.

The solution of the problem is determination of the optimum law of vector control change $U^{(\beta \beta)}\left(\varphi, l_{x}, l_{y}, l_{z}\right)=\left[q_{x}^{(\alpha)}\left(\varphi, l_{y}, l_{z}\right), q_{y}^{(\alpha \alpha)}\left(\varphi, l_{x}, l_{z}\right), q_{z}^{(\alpha)}\left(\varphi, l_{x}, l_{y}\right)\right], \quad \beta=1,2, \quad$ providing $J^{o p t}\left(q_{x(o p t)}^{(\alpha \alpha)} q_{y(o p t)}^{(\alpha \alpha)} q_{z(o p t)}^{(\alpha \alpha)}\right)=\min _{q_{x}^{(\alpha \alpha)}, q_{y}^{(\alpha \alpha)}, q_{z}^{(\alpha \alpha)}} J, J=\left\|\Theta\left(l_{x}, l_{y}^{*}, l_{z}^{*}, \varphi_{k}\right)\right\|_{L_{\infty}\left[l_{x} \in L\right]}$. at the specified value

\footnotetext{
* Corresponding author: entcom@samgtu.ru
} 
$\varphi=\varphi_{\mathrm{k}}$ - maximum accuracy task $(\beta=1)$ or $\min _{\mathrm{q}_{\mathrm{x}}^{(\alpha)}, \mathrm{q}_{\mathrm{y}}^{(\alpha)}, \mathrm{q}_{\mathrm{z}}^{(\alpha)}} \varphi_{\mathrm{k}}$ at the specified value $J=J_{\text {des }}$ maximum performance task $(\beta=2)$ in terms of restrictions on the allowable temperature $\Theta_{d}=$ const .

$$
\Theta(\rho, \varphi)=T(\rho, \varphi)-T_{\text {зад }} \leq \Theta_{d}, \varphi \in(0, \infty), \rho \in \bar{\Omega}_{\rho}
$$

and resource control:

$$
\begin{aligned}
& \left|q_{x}^{(\alpha \alpha)}\left(\varphi, l_{y}, l_{z}\right)\right| \leq 1 \quad ; \alpha=3,4 ; l_{x}=0 ; l_{x}=1 ; \\
& \left|q_{y}^{(\alpha \alpha)}\left(\varphi, l_{x}, l_{z}\right)\right| \leq 1 ; \alpha=5,6 ; l_{y}=0 ; l_{y}=l_{y_{\max }} ; \\
& \left|q_{z}^{(\alpha \alpha)}\left(\varphi, l_{x}, l_{y}\right)\right| \leq 1 ; \alpha=1,2 ; l_{z}=0 ; l_{z}=l_{z_{\max }} ;
\end{aligned}
$$

when exposed to a control object of the internal and external disturbances. $\varphi_{k}=\frac{\lambda \tau_{k}}{C x^{2}{ }_{\max }}$ the relative end time of the process, $\lambda, \mathrm{C}$ - thermal conductivity and specific heat of the material of construction. In this example (Fig. 1) the temperature of the inner surface of the outer shell of the supporting structure has a constant time value $T_{\text {out }}$. The temperature of the outer shell affects the temperature of the faces of the plate by radiant heat transfer. From opposite sides of the supporting structure has a rectangular area, S1 and S2, which, through radiant heat transfer affects the temperature of the environment. On the lateral surfaces of construction installed heat-emitting devices, occasionally included in the work and controlled heat sources 1,2,3,4,5,6 attached to the surface areas S1 and S2 on opposite faces $\alpha=3$ and $\alpha=4$ of the supporting construction.

A valid control $\left|U^{(\beta \beta)}\left(\varphi, l_{x}, l_{y}, l_{z}\right)\right| \leq 1$ in this example can be represented in a multiplicative form: $\quad q_{x}^{(\alpha \alpha)}\left(\varphi, l_{y}, l_{z}\right)=U_{x}^{(\alpha \alpha)}(\varphi) V_{x}^{(\alpha \alpha)}\left(l_{y}, l_{z}\right), \quad \alpha=3,4 ; \quad \alpha=1,2,5,6$, $q_{x}^{(\alpha \alpha)}\left(\varphi, l_{x}, l_{y}\right)=q^{(\alpha \alpha)}\left(\varphi, l_{x}, l_{y}\right) \equiv 0$, fixed in advance the spatial distribution $V_{x}^{(\alpha \alpha)}\left(l_{y}, l_{z}\right)$ of the controllable heat sources on two opposite faces $\alpha=3,4$ with a specified coordinate location $V_{x}^{(\alpha \alpha)}, \alpha=3,4$. Under these conditions, the optimal control of autonomous $\bar{U}^{(\beta \alpha)}(\varphi)$ groups of heat sources each $\alpha$-th face:

$$
\begin{gathered}
\bar{U}^{\beta \alpha}(\varphi)=\left[U_{x}^{(\beta 3)}(\varphi), U_{x}^{(\beta 4)}(\varphi), U_{y}^{(\beta 5)}(\varphi), U_{y}^{(\beta 6)}(\varphi), U_{z}^{(\beta 1)}(\varphi), U_{z}^{(\beta 2)}(\varphi)\right] \\
U_{o p t}^{(1 \alpha)}(\varphi)=\arg \min _{\bar{U}^{(1 \alpha)}(\varphi)} J ; U_{o p t}^{(2 \alpha)}(\varphi)=\arg \min _{\bar{U}^{(2 \alpha)}(\varphi)} \varphi_{k} ;
\end{gathered}
$$

in the problem of maximum precision $(\beta=1)$ and task performance $(\beta=2)$ using the method of moments $[2,3]$ it is possible to parameterize and to reduce to the problem of finding a finite number of parameters $\Delta_{\delta}^{(\mathrm{i})}, \delta=1,2, i ; i=1,2, . . J$; where $\Delta_{\delta}^{(\mathrm{i})}$ the duration $\delta$-th interval of constancy of the total number $\mathrm{i}$, where each vector $\bar{U}^{(\beta \alpha)}(\varphi)$ component alternately takes its maximum value at the level of the constraints (2). 


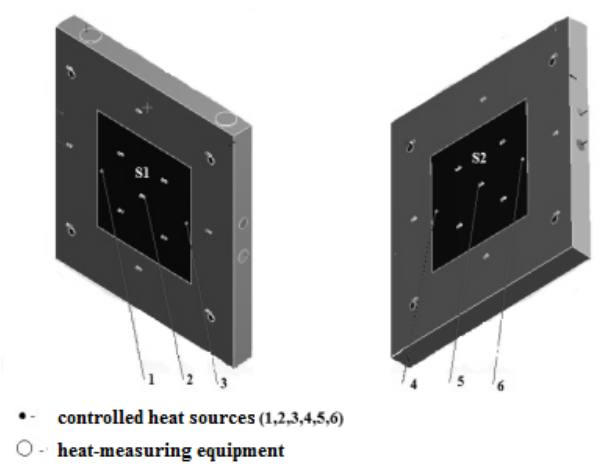

Fig. 1. The location of the heat sources on the supporting structure (example).

The duration $\Delta_{\delta}^{(\mathrm{i})}$ and the desired number of intervals $i$, is determined by the alternance method [3] from the system of transcendental equations [6]:

$$
\left.\Theta\left(\rho_{k}, \varphi_{k}\right)\right|_{\rho_{k} \in L_{k}}= \pm \varepsilon^{(i)},\left.\operatorname{grad} \Theta\left(\rho_{k}, \varphi_{k}\right)\right|_{\rho_{k} \in L_{k}}=0
$$

where $\left.\Theta\left(\rho_{k}, \varphi_{k}\right)\right|_{\rho_{k} \in L_{k}}$ - numerical solution of boundary value problems in the final time $\varphi_{k}$ points $\rho_{k}\left(l_{x}^{(k)}, l_{y}^{*}, l_{z}^{*}\right) \in L_{k}\left(l_{x}^{(k)}, l_{y}^{*}, l_{z}^{*}, \varphi_{k}\right) \subset L\left(l_{x}, l_{y}^{*}, l_{z}^{*}\right)$ of the considered line section $L\left(l_{x}, l_{y}^{*}, l_{z}^{*}\right)$, which constitute a countable set of limit points, which is the ratio (5).

The solution of the transcendental system (5) is carried out by a specially developed application package [5] based on the numerical solution of the boundary value problem in the ANSYS package. The processing of the results of the solution and its analysis were carried out using the developed application [5]. The results of calculations in the software package ANSYS are shown, which demonstrate the deviation $\Theta\left(L, \varphi_{k}\right)$ of the resulting temperature distribution $T\left(L, \varphi_{k}\right)$ from the given $T_{t a r}$ for different values of the thermal conductivity coefficient $\lambda$. Figure 2 shows the deviation $\Theta\left(L, \varphi_{k}\right)$ for the optimal control accuracy $\beta=1$ for 1 and 2 interval control in the responsible line $L\left(l_{x}, l_{y}^{*}, l_{z}^{*}\right) l_{y}^{*}=0,5$, $l_{z}{ }^{*}=0,5$ of the section of the load-bearing structure The maximum temperature deviation on this line $L\left(1_{\mathrm{x}}, 1_{\mathrm{y}}{ }^{*}, 1_{\mathrm{z}}{ }^{*}\right)$ does not exceed $4^{\circ} \mathrm{C}$ for a single-interval and $2^{\circ} \mathrm{C}$ for a twointerval temperature when exposed to the heaviest heat load option. 

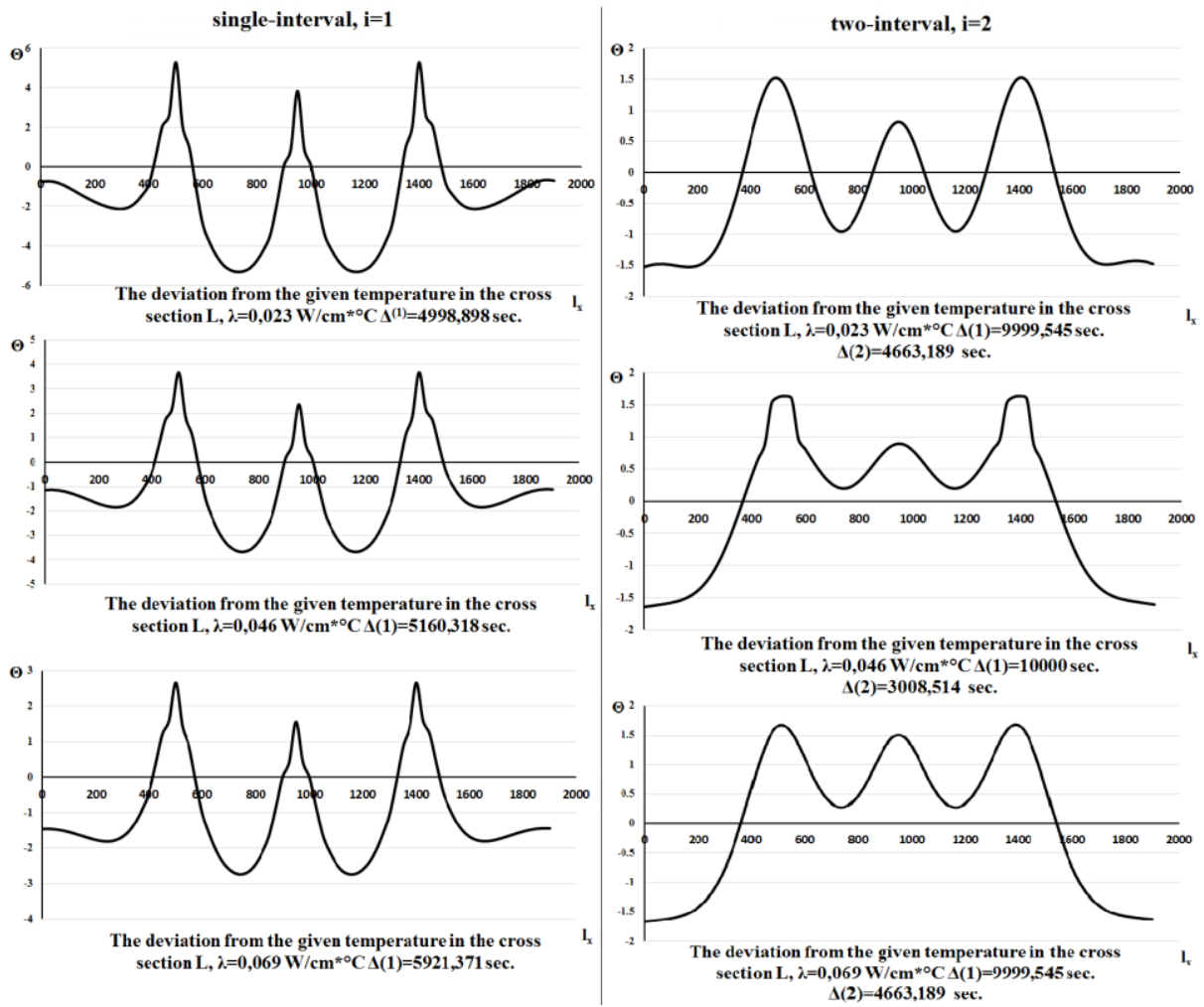

Fig. 2. Deviation from specified temperature on the section line $L\left(l_{x}, l_{y}{ }^{*}, l_{z}{ }^{*}\right), l_{y}{ }^{*}=0,5, l_{z}{ }^{*}=0,5$ with the optimal control accuracy.

The dependence of the optimization results on the properties of the construction material's thermal conductivity coefficient $\lambda$ is shown in Figures 3 and 4.

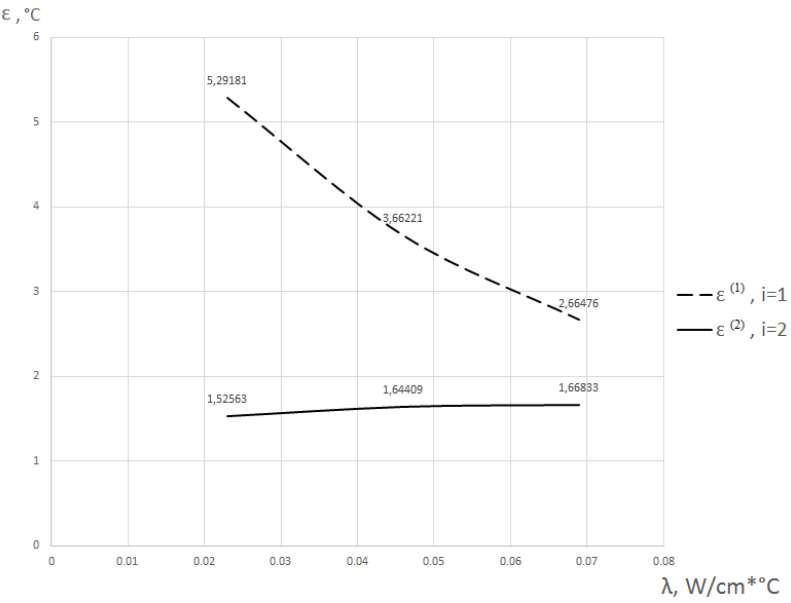

Fig. 3. The dependence of the accuracy of control on the value of thermal conductivity for single- and two-interval $(i=1, i=2)$ controls. 


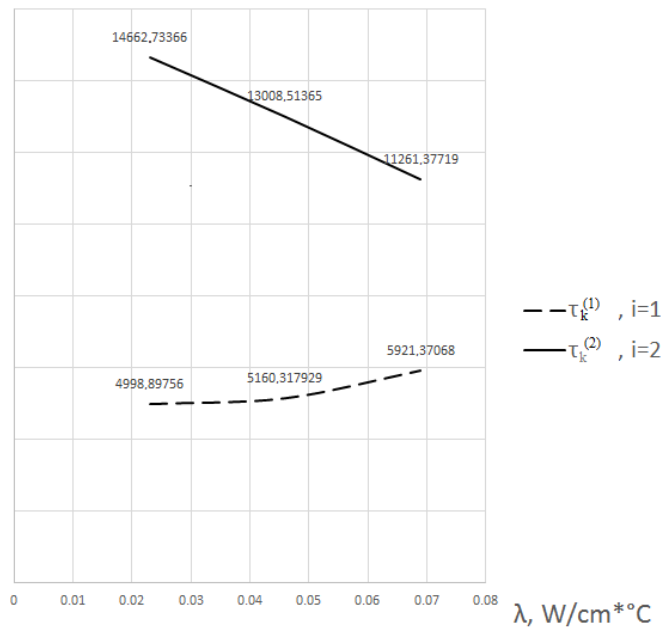

Fig. 4. The dependence of control time on the value of the coefficient of heat conductivity for singleand two-interval $(i=1, i=2)$ controls.

\section{Conclusions}

The analysis of the available parameters has shown that the two-interval $(i=2)$ control in both problems has a significantly lower sensitivity to the coefficient of thermal conductivity $\lambda$ with significantly higher accuracy of maintaining the set temperature. All this leads us to conclude that designer has greater freedom in choosing the material of the structure.

\section{References}

1. M.Y. Livshits, M.Y. Derevjanov, S.A. Kopytin, XIV Minsk International Forum on Heat and Mass Transfer: abstracts and reports, 1, 719 (2012)

2. A.G. Butkovskii, Theory of optimal control of distributed parameter systems (Nauka, Moscow, 1965) [In Russian]

3. E'.Ya. Rapoport, Optimal'noe upravlenie sistemami s raspredelenny'mi parametrami. (Vy'sshaya shkola, Moscow, 2009) [In Russian]

4. B.B. Borodulin, M.Y. Livshits, EPJ Web of Conferences, 110 (2016)

5. A.P. Efimov, Proc. VIII int. Conf. Samara scientific centre of RAS, 195 (2006)

6. B.B. Borodulin., M.Y. Livshits, S.E. Korshikov, MATEC Web Conf. 92, 26-28 (2016) 